\title{
Spectroscopy of HIP 113790 at the 5th OHP NEON summer school
}

\author{
Y. Frémat ${ }^{1}$, A. Antonova ${ }^{2}$, Y. Damerdji ${ }^{3}$, C.J. Hansen ${ }^{4}$, \\ M.T. Lederer ${ }^{5}$, M. Tüysüz ${ }^{6}$, P. Lampens ${ }^{1}$, P. Van Cauteren ${ }^{7}$ \\ ${ }^{1}$ Royal Observatory of Belgium, 3 avenue circulaire, 1180 Brussels, Belgium \\ ${ }^{2}$ Armagh Observatory, College Hill, Armagh, BT61 9DG, N. Ireland \\ ${ }^{3}$ Observatoire de Haute-Provence, 04870 St Michel l'Observatoire, France \\ ${ }^{4}$ N. Bohr Institute, Astronomy, Juliane Maries Vej 30, 2100 Copenhagen, Denmark \\ ${ }^{5}$ Institut für Astronomie, Türkenschanzstrasse 17, 1180 Wien, Austria \\ ${ }^{6}$ Department of Physics, Faculty of Arts and Sciences, Canakkale Onsekiz Mart \\ University, 17100, Canakkale, Turkey \\ 7 Beersel Hills Observatory (BHO), 1650 Beersel, Belgium
}

\begin{abstract}
In this contribution, we present the high-resolution observations we obtained for HIP 113790 at the Haute-Provence Observatory using the AURELIE spectrograph. By determing its fundamental parameters, we confirm that the star is located in the $\delta$ Scuti instability strip. We further report the detection of rapid line profile variations with a period of about 1.43 hours.
\end{abstract}

\section{Introduction and Observations}

HIP 113790 is a bright $(\mathrm{V}=7.30)$ poorly studied $\delta$ Scuti star (Frémat et al. 2006a) that shows radial velocity variations (Grenier et al. 1999) and that was classified "unsolved variable" in the HIPPARCOS catalogue (ESA 1997). Preliminary photometric observations (Frémat et al. 2005) were carried out in December 2004 and showed a complex multiperiodic variation pattern with a main period of about 1.2 hours. Taking advantage of the observing time available during the $5^{\text {th }}$ NEON school at the Observatoire de Haute Provence (OHP), we obtained time series of high-resolution spectra for 3 nights on the $1.52 \mathrm{~m}$ telescope equipped with the AURELIE spectrograph (Gillet et al. 1994). The observations log is given in Table 1 with Date (col.1), grating number (col.2), 
spectral range (col.3), resolution (col.4), time exposure (col.5) and number of exposures (col.6). Our aim was twofold: to derive the stellar parameters of the star and to interpret the radial velocity variability in terms of line profile variations.

Table 1: Description of the spectroscopic observations.

\begin{tabular}{lccrrr}
\hline Date & Grating & Spectral range & $\mathrm{R}$ & $\mathrm{t}(\mathrm{s})$ & $\mathrm{n}$ \\
\hline July 26, 2006 & 3 & $4000-4430$ & 5500 & 1200 & 5 \\
July 28, 2006 & 7 & $5380-5520$ & 28000 & 1200 & 14 \\
July 30, 2006 & 7 & $5380-5520$ & 28000 & 1200 & 12 \\
\hline
\end{tabular}

\section{Stellar parameters}

The stellar parameters of HIP 113790 were derived by comparing the observed $4000-4430 \AA$ wavelength range to synthetic spectra. Model atmosperes we used were computed by Castelli \& Kurucz (2003) with the ATLAS9 program and the theoretical spectra were obtained with the SYNSPEC (Hubeny \& Lanz 1995, see references therin) computer code for a solar-like chemical composition and a $2 \mathrm{~km} \mathrm{~s}^{-1}$ microturbulent velocity. A least-squares algorithm (Frémat et al. 2006b) was used to derive, first, the projected rotation velocity $(V \sin i)$ and the heliocentric radial velocity (RV) by fitting several metallic lines, then the effective temperature $\left(\mathrm{T}_{\text {eff }}\right)$ and surface gravity $(\log g)$ by studying the $\mathrm{H} \gamma$ and $\mathrm{H} \delta$ line profiles. Our results are as follows:

$$
\begin{aligned}
V \sin i & =28 \pm 3 \mathrm{~km} \mathrm{~s}^{-1} \\
\mathrm{RV} & =5.3 \pm 0.74 \mathrm{~km} \mathrm{~s}^{-1} \\
\mathrm{~T}_{\mathrm{eff}} & =7185 \pm 100 \mathrm{~K} \\
\log g & =3.80 \pm 0.12 .
\end{aligned}
$$

The averaged radial velocity we obtain is similar to the value $(R V=5.8 \pm$ $7.6 \mathrm{~km} \mathrm{~s}^{-1}$ ) published by Grenier et al. (1999), and there is therefore no sign of multiplicity. We further noticed no chemical peculiarity in the spectra of HIP 113790 and we obtained a very good agreement between observations and theory as shown in Fig. 1. However, since the effective temperature of the star is close to the limit at which the hydrogen lines become less sensitive to surface gravity, we also estimated its $\log g_{\pi}$ by computing the luminosity, from the $\mathrm{V}$ magnitude and the trigonometric parallax, and by interpolating the stellar mass in theoretical evolutionary tracks (Schaller et al. 1992). The value we obtained $\left(\log g_{\pi}=3.89 \pm 0.09\right)$ is in fair agreement with the value derived from spectroscopy. 


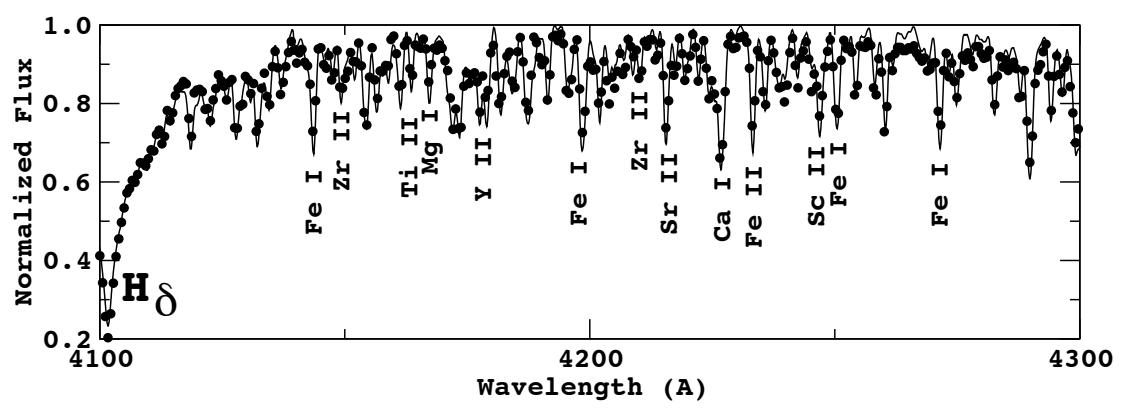

Figure 1: Comparison between observations (full line) and synthetic spectrum (dots).

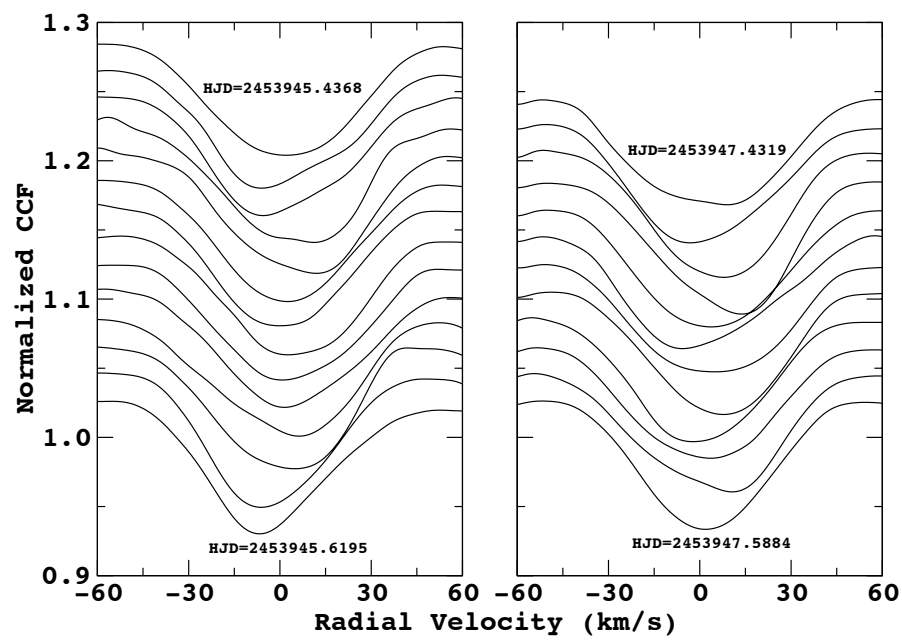

Figure 2: Variation of the cross-correlation function during two nights.

\section{Variability: Spectroscopy}

We performed a preliminary study of the spectroscopic variations of HIP 113790 in a spectral domain ranging from 5380 to $5520 \AA \AA$. In order to increase the S/N ratio of the observations, each exposure was cross-correlated with a synthetic spectrum computed adopting the $\mathrm{T}_{\text {eff }}$ and the $\log g$ values we previously derived. The time series of the resulting cross-correlation functions (CCF) are plotted in Fig. 2 for two nights and clearly show rapid spectroscopic variations with a period of about 1.43 hours, as we determined from the study of the residuals at the line center using the PERIOD04 software (Lenz \& Breger 2005). 


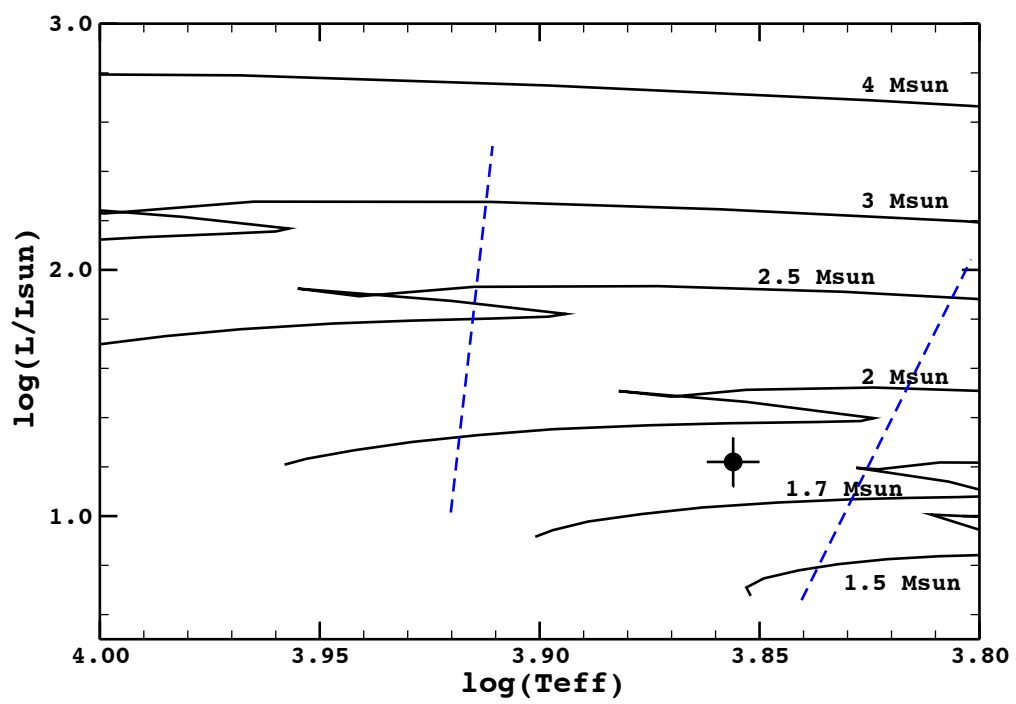

Figure 3: Location of HIP 113790 in the HR diagram. Broken lines represent the expected borders of the $\delta$ Scuti instability strip (Dupret et al. 2005). Evolutionary tracks (full lines) are from Schaller et al. (1992).

\section{Conclusions}

As shown by the stellar parameters we derived, HIP 113790 is located in the $\delta$ Scuti instability strip (Fig. 3). From the study of our cross-correlation function time series, the radial velocity variability can definitively be interpreted as due to periodic line profile variations, with a main period (i.e. 1.4 hours) of the same order as the variations detected in the photometry (i.e. 1.2 hours). Additional spectroscopic observations would however be required on a larger telescope to perfom a complete frequency analysis on several individual spectral lines. During the past year, an extensive photometric follow-up of HIP 113790 was further carried out using the $\mathrm{V}$ and $\mathrm{B}$ filters at different observatories in Belgium (P.Lampens \& P.Van Cauteren), in Greece (K.D.Gazeas \& P.G.Niarchos, S.Klides) and in the USA (C.W.Robertson). A full analysis of these data is presently undertaken.

Acknowledgments. We thank the OPTICON programme, M.Dennefeld and D.Gillet for funding our participation to the NEON summer school. Financial support from the Belgian Federal Science Policy is gratefully acknowledged (projects IAP P5/36 and $\mathrm{MO} / 33 / 018$ ). 


\section{References}

Castelli, F., \& Kurucz, R. 2003, IAU Symposium 210, 20

ESA 1997, The Hipparcos and Tycho Catalogues ESA-SP 1200

Frémat, Y., Lampens, P., Van Cauteren, P., \& Robertson, C.W. 2005, CoAst, 146, 6

Frémat, Y., Lampens, P., Van Cauteren, P., \& Robertson, C.W. 2006a, Mm.S.A.It., 77,174

Frémat, Y., Neiner, C., Hubert, A.M., et al. 2006b, A\&A, 451, 1053

Gillet, D., Burnage, R., Kohler, D., et al. 1994, A\&AS, 108, 181

Grenier, S., Baylac M.O., Rolland L., et al. 1999, A\&AS, 137, 451

Hubeny, I., \& Lanz, T. 1995, ApJ, 439, 875

Lenz, P., \& Breger, M. 2005, CoAst, 146, 53

Schaller, G., Schaerer, D., Meynet, G., \& Maeder, A. 1992, A\&AS, 96, 269 
